\title{
Ciclo de vida y enemigos naturales de Mechanitis menapis (Lepidoptera: Ithomiini)
}

\author{
Paola, G. Santacruz ${ }^{1 *}$, Emma, Despland ${ }^{2} \&$ Carlos E Giraldo ${ }^{3}$ \\ 1. Museo Interactivo de Ciencia, Tababela Oe1-60 y Antonio de Latorre, Quito, Ecuador; \\ paolasantacruzendara@gmail.com \\ 2. Professor, Biology Department, Concordia University. Quebec. Canadá; emma.despland@concordia.ca \\ 3. Grupo de Investigación de Sanidad Vegetal. Facultad de Ciencias Agropecuarias, Universidad Católica de Oriente. \\ Sector 3, cra. 46 No. 40B 50, Rionegro, Antioquia-Colombia; cegiral0@gmail.com \\ * Correspondencia
}

Recibido 08-IV-2019. Corregido 12-VI-2019. Aceptado 30-IX-2019.

\begin{abstract}
Life cycle and natural enemies of Mechanitis menapis (Lepidoptera: Ithomiini). The butterflies of the Ithomiini tribe are one of the most-studied biological models of recent years in terms of biogeography, taxonomy, and evolution. However, even though their biology and distribution is better known than many other groups of butterflies, there are unknown aspects of their natural history that would improve our understanding of their behavior, population dynamics, and interactions with their environment. In this work, we studied the natural history of the butterfly Mechanitis menapis mantineus Hewitson (Nymphalidae: Ithomiini), and its natural enemies, in Western Ecuador. We identified three host plants, which are new records for the species in this region. We documented the life cycle and described the morphology of the immature stages, their development time, and studied the factors associated with mortality of these immature stages. Additionally, we identified the parasitoids associated with the species in the study area. In particular, we documented aspects of the natural history and behavior of Hyposoter sp. (Ichneumonidae: Campopleginae), the main parasitoid of the larvae. The results of this work provide essential information for the taxonomic revision of the genus Mechanitis F. as well as the continued study of the interactions between the butterfly, its host plants, and natural enemies.
\end{abstract}

Key words: life history, Solanaceae, host plant, Nymphalidae, Ecuador, Andes.

Santacruz, P. G., Despland, E., \& Giraldo, C. E. (2019). Ciclo de vida y enemigos naturales de Mechanitis menapis (Lepidoptera: Ithomiini). Revista de Biología Tropical, 67(6), 1488-1504.

Las mariposas Ithomiini (Lepidoptera: Nymphalidae) son un grupo de distribución exclusivamente neotropical, que ha encontrado su máxima diversificación en los valles interandinos y piedemonte amazónico asociada a la rápida elevación de los Andes (Elias et al., 2009; De-Silva et al., 2010; De-Silva, Elias, Willmott, Mallet, \& Day, 2015; GarzónOrduña, Silva-Brandão, Willmott, Freitas, \& Brower, 2015; De-Silva et al., 2017; Chazot et al., 2016). Su riqueza supera las 370 especies y más de 1500 subespecies que pueden encontrarse principalmente en bosques, desde el nivel del mar hasta los $3000 \mathrm{~m}$ de altura (Lamas, 2004; Willmott \& Freitas, 2006). La tribu Ithomiini se puede reconocer morfológicamente por la presencia de mechones eréctiles ubicados en el margen anterior de las alas posteriores de los machos (Fox, 1940). Sus estados inmaduros han sido relativamente bien estudiados en comparación con otros grupos de Nymphalidae (Brown \& Freitas, 1994), 
no obstante, aún es escasa la información sobre las historias de vida y estados inmaduros de especies Andinas (Freitas \& Brown, 2008).

Los estados inmaduros de la tribu Ithomiini, presentan una fuerte asociación con sus plantas hospederas pertenecientes principalmente a la familia Solanaceae, y aunque no se ha encontrado evidencia clara de coevolución tradicional o de cladogénesis de herbívoros y sus hospederos, si existe una fuerte correlación entre la diversidad de los clados de la familia Solanaceae y los clados de Ithomiini que se especializan en aquellas plantas (Willmott \& Freitas, 2006; Garzón-Orduña et al., 2015). Por esta razón y dado que conforman diferentes anillos miméticos que pueden coexistir en un mismo microhábitat, las especies de esta tribu han servido como modelo en investigaciones de ecología química, evolución y biogeografía (Brown, 1979; Brown, Jr., 1987; Joron \& Mallet, 1998; Willmott \& Mallet, 2004; Elias, Gompert, Jiggins, \& Willmott, 2008).

Uno de los géneros de Ithomiini que más interés ha despertado es el género Mechanitis $F$. Estas mariposas, generalmente reconocidas como "bandas de tigre" forman parte de complejos miméticos tanto Batesianos como Mullerianos (Brown \& Benson 1974; Brown, Jr., 1977) y exhiben una exuberancia genética y morfológica, que las hace un modelo evolutivo único para estudiar (Fox, 1967; Brown, Jr., 1977; Giraldo \& Uribe, 2012; Hill et al., 2012). No obstante, esta gran diversidad y variación en el género ha generado cambios taxonómicos frecuentes (Fox, 1967; Brown, Jr., 1977, Lamas, 2004; Hill et al., 2012) y es sujeto actualmente de revisión taxonómica (Giraldo in prep). En la clasificación vigente se reconocen cinco especies y 47 subespecies (Lamas, 2004; Hill et al., 2012). Para cada una de sus especies, se han identificado plantas hospedantes y descrito ciclos de vida (Giraldo \& Uribe, 2010a; Hill et al., 2012; Giraldo, Ramírez-Restrepo, Escobar, \& Uribe, 2014), sin embargo, la relación particular de las diferentes subespecies con sus plantas hospedantes de uso regionales y la relación con sus enemigos naturales continúan siendo desconocidas.
Para Mechanitis menapis (Hewitson, 1856) se conocen particularmente las plantas hospederas y los ciclos de vida de las subespecies presentes en Centroamérica: Mechanitis menapis doryssus Bates 1864, en México (Giraldo et al., 2014); Mechanitis menapis saturata Godman (1901), en Costa Rica (Drummond \& Brown, 1987) y Mechanitis menapis occasiva (Fox, 1967) en los valles interandinos colombianos (Giraldo \& Uribe 2010b), sin embargo poco se conoce sobre las plantas hospederas y los ciclos biológicos de la subespecie de la vertiente del pacífico ecuatoriano Mechanitis menapis mantineus (Hewitson, 1869). Datos moleculares presentados en trabajos recientes, sugieren que esta subespecie merecería el estatus de especie, dada las distancias genéticas que presenta con taxones relacionados (Dasmahapatra, Elias, Hill, Hoffman, \& Mallet, 2010; Giraldo \& Uribe 2012; Hill et al., 2012); de ser así, este taxón podría ser considerado un grupo endémico para la región del oeste de Ecuador y cualquier información biológica relacionada al mismo es de suma importancia en términos ecológicos y evolutivos.

Este trabajo tuvo como objetivos, identificar las plantas hospederas, describir el ciclo biológico e identificar las causas principales de mortalidad de los estados inmaduros en condiciones silvestres de M. menapis mantineus, en el Valle de Mindo Pichincha - Ecuador. Adicionalmente, se describieron aspectos de la historia natural y el comportamiento del principal parasitoide de larvas en condiciones naturales. Los resultados de este trabajo, proporcionan información esencial para la revisión taxonómica del género Mechanitis y una línea base para continuar el estudio de la interacción de esta especie con sus plantas hospederas y enemigos naturales.

\section{MATERIALES Y MÉTODOS}

Localización: El trabajo fue desarrollado en la región occidental de la provincia de Pichincha-Ecuador, en el Valle de Mindo $\left(00^{\circ} 03\right.$ '44.1” S \& 7845'41.7” W; $\left.1390 \mathrm{~m}\right)$ (Fig. 1). Mindo, hace parte del Sistema 
Nacional de Áreas Protegidas, que cubre una franja continua de vegetación que va desde los 1180 a los 4780 m en la cima del volcán Guagua Pichincha y está en el límite inferior occidental de Bosque Protector Mindo-Nambillo. $\mathrm{Su}$ zona de vida hace parte de los bosques de neblina montanos de la Cordillera Occidental según Sierra et al. (1999). Los datos biológicos se han recopilado entre el 2013 y 2017.

Material biológico y cría: Los estados inmaduros fueron recolectados en campo sobre diferentes plantas hospederas, de las cuales se tomaron muestras botánicas para su posterior identificación en el herbario QCA de la Pontificia Universidad Católica del Ecuador. Los individuos se transportaron al laboratorio y se criaron en recipientes plásticos bajo condiciones controladas de temperatura y humedad $\left(21{ }^{\circ} \mathrm{C} ; 50 \%\right.$ humedad relativa; $\left.12 \mathrm{~h} \mathrm{luz}\right)$. Las larvas se alimentaron con hojas de las plantas hospederas hidratadas y dispuestas a voluntad de consumo. Se realizaron observaciones diarias para identificar cambios de estadio y obtener medidas de la longitud de las larvas y la amplitud de su cápsula cefálica bajo un estereomicroscopio. Las mediciones de los estados inmaduros siguieron lo sugerido por Freitas y Brown Jr. (2008) y las descripciones morfológicas de las larvas se realizaron siguiendo a Stehr (1987).

Tasas de desarrollo y fuente de alimentación: En este experimento fueron criadas siete larvas en siete hospederos distintos Solanum quitoense Lam., Solanum betaceum Cav., y Solanum acerifolium Dunal. Se realizaron tres réplicas en cada uno de ellas (n $=63$ ). Durante este período, el desarrollo de las orugas fue medido de manera similar a la mencionada anteriormente. Se usaron plantas hospederas de un año de edad y estado fenológico similar, para la alimentación de las larvas. La tasa de desarrollo fue estudiada usando dos

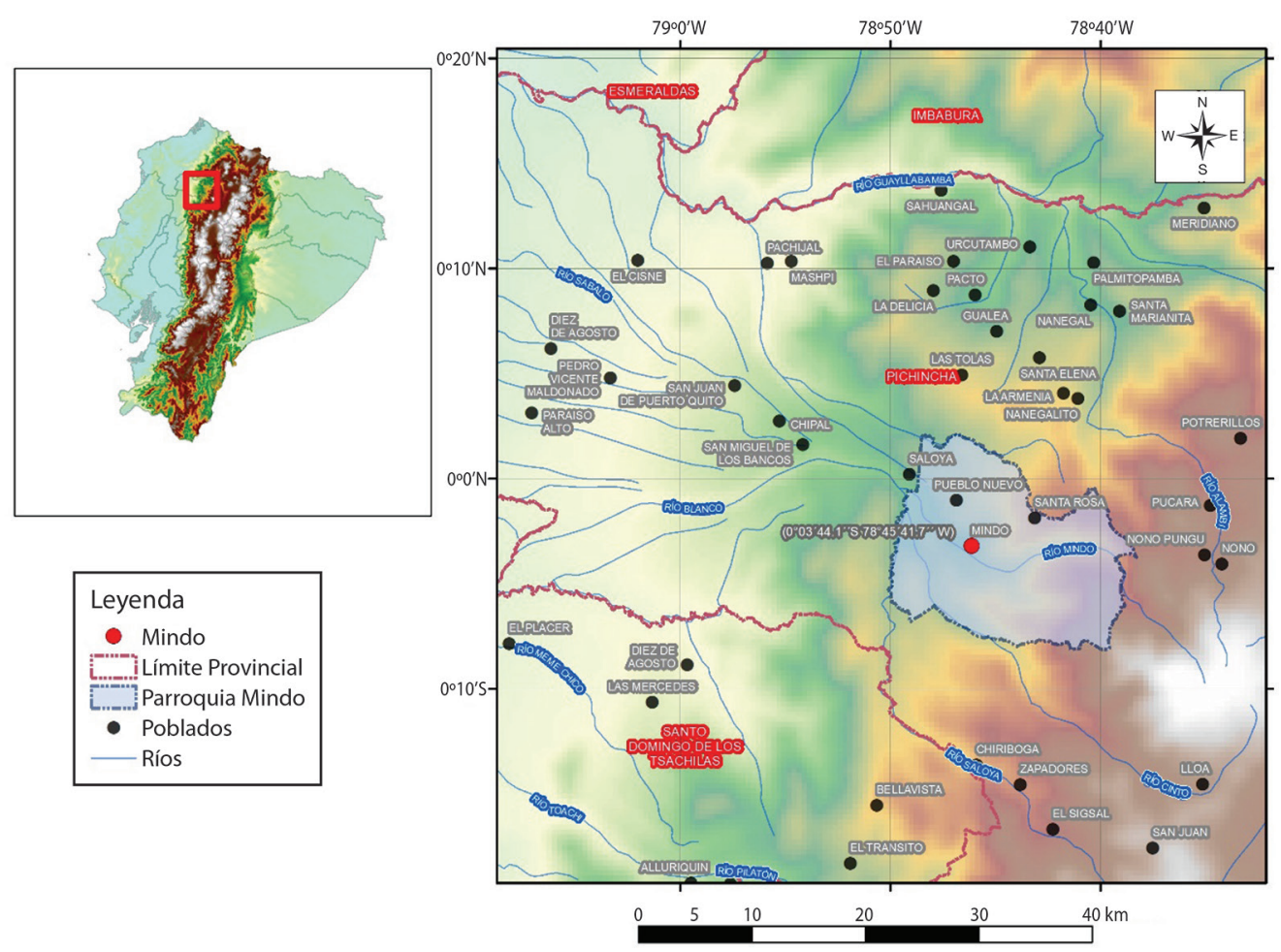

Fig. 1. Zona de estudio.

Fig. 1. Location of the study area. 
indicadores: 1) la longitud del cuerpo de las orugas, y 2) la medida de las cápsulas cefálicas. La longitud del cuerpo se analizó usando un ANOVA jerárquico a tres niveles no-equilibrado para cada uno de los estadios por separado. Las variables grupales fueron, en cada caso, la especie hospedera y el número de plantas y la variable dependiente fue un promedio de la longitud de los tres individuos criados en cada planta. Las medidas de las cápsulas cefálicas fueron analizadas con un ANOVA de un factor DCA con tamaño desigual de muestras para cada estadio por separado. La variable dependiente fue el ancho de las cápsulas cefálicas y el factor independiente fueron las especies.

Agentes de mortalidad en campo: Se diseñó un experimento para estudiar los agentes causales de mortalidad y los índices de mortalidad de las larvas en campo, usando dos cohortes de diez individuos, durante dos épocas climáticas, seca y lluviosa $(\mathrm{n}=40)$. Se evaluaron los efectos de la planta hospedera y las dos épocas climáticas en los índices de mortalidad. Las especies de plantas hospederas usadas para dicho experimento ( $S$. betaceum y $S$. acerifolium), fueron criadas de manera controlada y posteriormente dispuestas en campo para el experimento. Se realizaron observaciones diarias durante el período de desarrollo de los individuos desde huevo hasta la emergencia de los adultos. Para analizar los datos, se usó el test de probabilidad exacta de Fisher, usando como categoría excluyente, si la larva estaba viva o muerta, y como grupos las dos especies de planta hospedera. Se construyeron curvas de supervivencia por planta y por época climática. La causa posible de la muerte, o agente causal, y el estadio larval de cada individuo fueron especificados y las larvas con evidencia de parasitoides fueron recolectadas y se conservaron en el laboratorio hasta la obtención de los parasitoides adultos. Los parasitoides adultos se preservaron en alcohol al $70 \%$, para su posterior identificación a nivel de familia.

\section{Comportamiento del parasitoide Hypo- soter sp. (Ichneumonidae)}

Cortejo y cópula: Los parasitoides fueron criados en orugas de Mechanitis menapis mantineus de cuarto estadio por más de 15 generaciones en condiciones de laboratorio, según el procedimiento descrito por Fatouros et al. (2005). Para observar el comportamiento de cortejo y cópula, se dispusieron 50 parejas, de cinco a siete días de emergencia, por $24 \mathrm{~h}$ en recipientes de vidrio. Los individuos fueron alimentados con una solución de miel y agua (1:1). Las parejas de parasitoides, fueron observadas por el lapso de tres horas diarias durante un mes, obteniendo etogramas de cortejo, determinados por los movimientos observados.

Búsqueda y oviposición: La observación de oviposición del parasitoide, se realizó dentro de cajas de vidrio, donde se dispuso la hembra del parasitoide, y una larva en cuarto estadio sobre su planta hospedera. Los ensayos se realizaron con 50 hembras y se observaron durante tres horas diarias por un mes. Posteriormente se elaboraron etogramas del cortejo y la oviposición.

Para conocer el grado de estímulo de las emisiones volátiles de $M$. menapis mantineus hacia los parasitoides se realizaron tres ensayos: 1) con el estímulo del adulto, 2) con el estímulo usando excremento de oruga, 3 ) con el estímulo mecánico de la presencia de la oruga.

Para el primer ensayo, las plantas fueron colocadas por $12 \mathrm{~h}$ en una jaula cilíndrica de tela ( $1 \mathrm{~m}$ x $50 \mathrm{~cm}$ de diámetro), con 20 adultos, propiciando la oviposición de las mariposas. Para los dos experimentos siguientes, se emplearon hojas hidratadas de las dos plantas hospederas seleccionadas ( $S$. betaceum y $S$. acerifolium) cortadas en cuadrados de $2 \times 2$ $\mathrm{cm}$, según la metodología descrita por Fatouros et al. (2005). En el segundo ensayo, fueron utilizadas hojas de las plantas hospedantes y excremento de orugas; en el tercer ensayo, fueron colocadas orugas de cuarto estadio sobre las plantas hospederas. 
Para reconocer la respuesta al estímulo en ambas especies de planta, se usaron 10 avispas dispuestas individualmente en cajas Petri, donde se encontraban, ambas especies de plantas y cada especie por separado, para un total de 30 réplicas por experimento.

A cada una de las avispas se les ofreció simultáneamente un cuadrado de la hoja, con cada ensayo respectivo. El total del tiempo utilizado por la avispa, dentro de la caja Petri fue de 10 minutos. Cuando la avispa se acercaba a uno de los cuadrados era registrado como una respuesta positiva al estímulo. Cada avispa fue usada una sola vez y luego descartada.

Para analizar los datos se usó el Test de probabilidad exacta de Fisher, usando como categoría excluyente si la avispa mostraba una respuesta positiva o negativa al estímulo y como grupos las diferentes especies de planta hospedera.

\section{RESULTADOS}

Historia natural: Se identificaron tres especies de plantas nutricias para las orugas de M. menapis mantineus en la zona de estudio: Solanum acerifolium Dunal., S. myriacanthum Dunal. y $S$. quitoense Lam. Sin embargo, ninguno de los individuos criados en condiciones de laboratorio sobre esta última planta, alcanzó el estado adulto. Por otra parte, bajo condiciones controladas, se observaron también oviposiciones en $S$. betaceum, planta cultivada no encontrada en forma silvestre en la región, por lo cual se usó en los experimentos de agentes de mortalidad en campo. La mayor parte de los estados inmaduros se recolectaron en plantas de $S$. acerifolium (60 \%) (Fig. 2), y en menor proporción en $S$. myriacanthum (39\%) y $S$. quitoense (1\%).

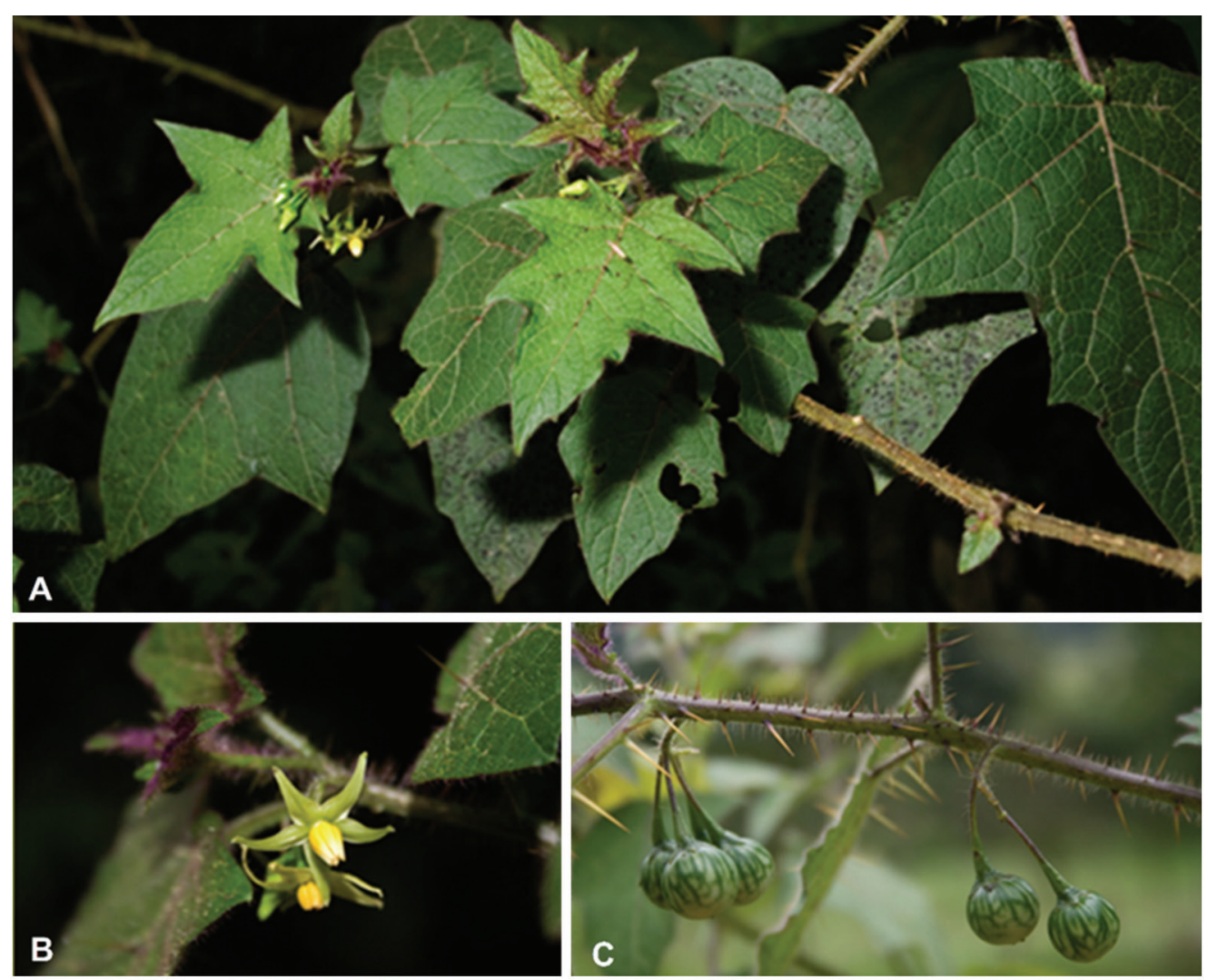

Fig. 2. Solanum acerifolium, planta hospedera de Mechanitis menapis mantineus. A. hojas, B. flores, C. frutos. Fig. 2. Solanum acerifolium, Host plan of Mechanitis menapis mantineus. A: leaves, B: flowers, C: fruits. 
Después de la eclosión, las larvas devoran el corión y poco tiempo después comienzan a alimentarse de la epidermis de las hojas de la planta hospedera; luego de unas pocas horas, se desplazan al envés de la hoja donde continúan su alimentación hasta el último estadio larval. En condiciones silvestres, se encontraron grupos de seis hasta 15 larvas en una misma hoja y fue común localizarlas en grupos mixtos de varios estadios larvales. Se observaron desplazamientos de forma gregaria de una hoja a otra, en la medida que las hojas fueron consumidas totalmente. Dicho comportamiento es mantenido hasta el cuarto estadio. Las larvas, se ubicaron comúnmente en las hojas de la parte media de la planta, y en muy raras ocasiones en el envés de las hojas de la parte apical. En condiciones de cría, se observó que todos los estadios larvales tienen hábitos diurnos y que el periodo de reposo ocurre en horas de la noche, cerca de las nervaduras de las hojas. El proceso de muda, ocurrió de forma sincronizada, en cada uno de los cambios de estadio larval. Al alcanzar su talla máxima en el quinto estadio la larva se torna amarilla. La prepupa asume forma de gancho o "J" justo antes de formar la crisálida. El proceso de empupamiento ocurre frecuentemente en el envés de las hojas en condiciones silvestres, donde se encontraron grupos hasta de cinco individuos.

Huevos: Tiempo de desarrollo: 6 días promedio $(\mathrm{n}=63)$. Los huevos se encontraron siempre en el haz de las hojas, normalmente en el tercio medio de la planta. El $64.3 \%$ (n = 63) de las posturas se encontraron de manera individual y el $15.9 \%(n=65)$ en pares. El 97 $\%$ de las posturas se encontraron en grupos no mayores de 6 huevos y una sola postura de 15 huevos sobre $S$. acerifolium. Los huevos tienen forma de barril alargado (Fig. 3A), $1.34 \mathrm{~mm}$ de longitud y 0.74 de diámetro, con un radio medio (longitud: amplitud) de $1.8(\mathrm{n}=4)$. Son de coloración blanca, con surcos longitudinales y 13 costillas longitudinales prominentes.

Primer estadio: Tiempo de desarrollo: 3 días $(\mathrm{n}=42)$; amplitud promedio de cápsula cefálica: $0.3 \mathrm{~mm}(\mathrm{n}=63)$; longitud corporal máxima: $3 \mathrm{~mm}$ (Fig. 3B). Cápsula cefálica negra y cuerpo blanco; una vez comienza su alimentación en la planta, se torna verde oscuro. Las pseudopatas son blancas sin evidencia de placas laterales y los ornamentos abdominales son cortos y poco visibles, sin coloración diferente al cuerpo.

Segundo estadio: Tiempo de desarrollo: 7 días $(\mathrm{n}=42)$; amplitud promedio de cápsula cefálica: $0.6 \mathrm{~mm}(\mathrm{n}=42)$; longitud corporal máxima: $6 \mathrm{~mm}$. Cápsula cefálica negra y cuerpo verde oscuro; pseudopatas de aspecto similares al primer estadio; los ornamentos laterales del primer y segundo segmento abdominal, son más largos y de color amarillo en la base, distinguibles de los demás que continúan blancos hasta el siguiente estadio.

Tercer estadio: Tiempo de desarrollo: 7 días $(\mathrm{n}=42)$; amplitud promedio de cápsula cefálica: $0.8 \mathrm{~mm}(\mathrm{n}=42)$; longitud corporal máxima: $12 \mathrm{~mm}$. (Fig. 3C). El cuerpo se torna color verde pálido y su cápsula cefálica es de color café oscuro; las pseudopatas son de aspecto similar al estadio anterior. El aspecto de los ornamentos es similar al del segundo estadio, sin embargo, un par de ornamentos en el pronoto se hacen visibles, aunque más cortos que los ornamentos abdominales (Fig. 3D).

Cuarto estadio: Tiempo de desarrollo: 7 días $(\mathrm{n}=42)$; amplitud promedio de cápsula cefálica: $1.2 \mathrm{~mm}(\mathrm{n}=42)$; longitud corporal máxima: $25 \mathrm{~mm}$ (Fig. 3E). La cápsula cefálica se torna gris al igual que el resto del cuerpo; las pseudopatas son de aspecto similar al estadio anterior. Todos los ornamentos se tornan amarillos en la base y blanquecinos en el extremo distal.

Quinto estadio: Tiempo de desarrollo: 7 días $(\mathrm{n}=42)$; amplitud promedio de cápsula cefálica: $1.8 \mathrm{~mm}(\mathrm{n}=42)$; longitud corporal máxima: $18 \mathrm{~mm}$ (Fig. 3F). La coloración de la cápsula cefálica se hace más blanquecina que la del cuarto estadio y su coloración corporal es 

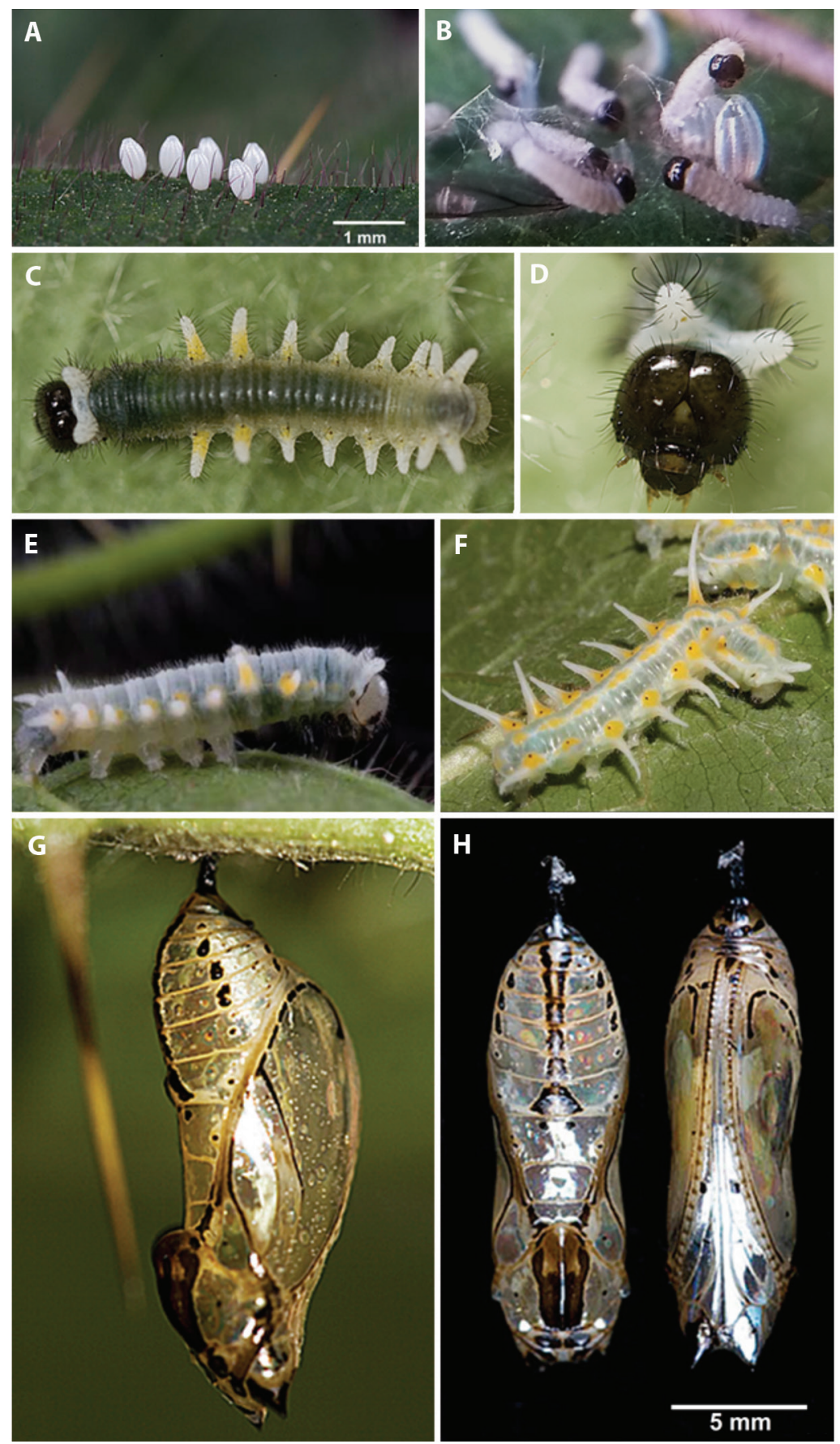

Fig. 3. Estados immaduros de Mechanitis menapis mantineus. Huevos (A); Primer estadio (B); Tercer estadio (C y D); Cuarto estadio (E); Quinto estadio (F); Pupa (G y H).

Fig. 3. Immature stages of Mechanitis menapis mantineus. Eggs (A); First larval instar (B); Third larval instar (C y D); Fourth larval instar (E); Fifth larval instar (F); Pupa (G y H). 


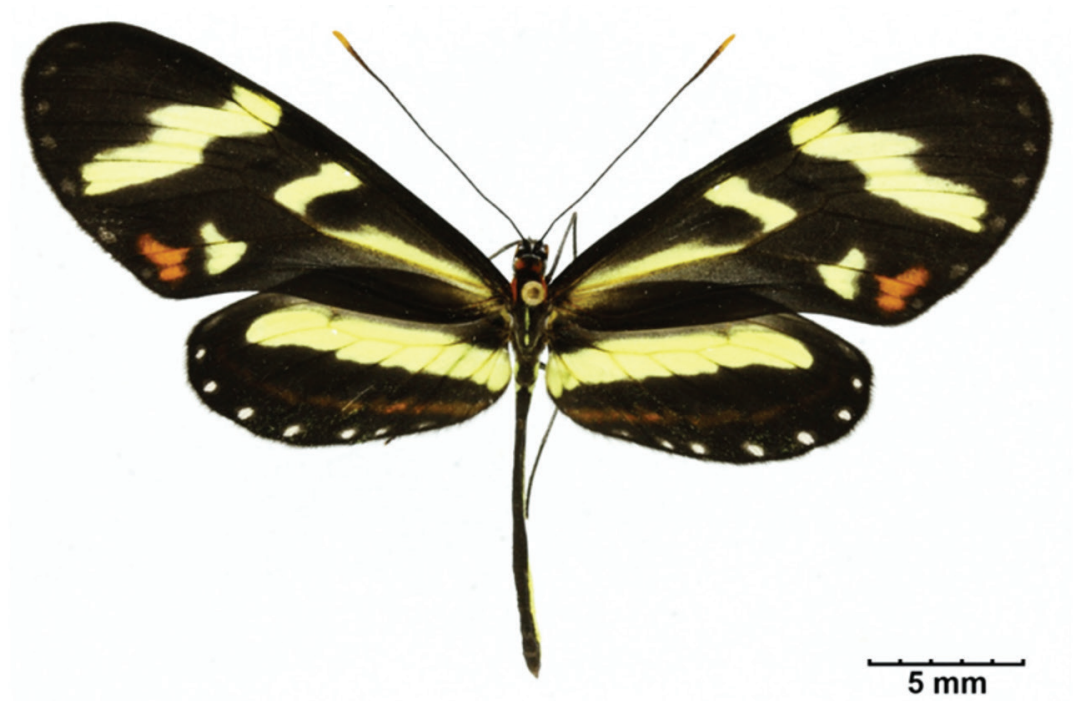

Fig. 4. Adulto de Mechanitis menapis mantineus.

Fig. 4. Adult of Mechanitis menapis mantineus.

más intensa, al igual que la de los ornamentos. La prepupa es de coloración amarillo intenso y ocurre aproximadamente unas pocas horas antes de empupar.

Pupa: Tiempo de desarrollo: 11 días ( $\mathrm{n}=$ 42); amplitud lateral $6.4 \mathrm{~mm}$; amplitud ventral $4.7 \mathrm{~mm}$; longitud $17 \mathrm{~mm}(\mathrm{n}=42)$ (Fig. 3G, Fig. $3 \mathrm{H})$. La pupa es de color dorado, con marcas negras discontinuas que denotan los apéndices alares del individuo en formación. El cremaster es negro y levemente curvado ventralmente. Antes de la emergencia del adulto, la pupa se torna oscura y permite diferenciar máculas del patrón alar del adulto.

Adulto: Los adultos de ambos sexos son similares en tamaño, con una longitud promedio del ala anterior de $34 \mathrm{~mm}$; patrón de coloración alar sin dimorfismo sexual evidente. (Fig. 4). La emergencia ocurre de manera simultánea en la mayoría de los casos, en horas de la mañana. Relación sexual es 1:1.

Tasas de desarrollo en diferentes plantas: La especie $S$. quitoense fue excluida del análisis debido a la alta tasa de mortalidad de las orugas observadas sobre la misma. Por este motivo, las comparaciones entre las tasas de desarrollo se realizaron sobre la especie $S$. acerifolium (utilizada en forma silvestre) y $S$. betaceum (usada sólo en laboratorio), no obstante, no se encontraron diferencias significativas en las tasas de crecimiento comparadas (Fig. 5).

Agentes de mortalidad: El experimento en campo mostró una mortalidad total del $60 \%$ (24/40), siendo mayor en la época seca (35\%) que en la época lluvia (25\%). De igual manera, fue mayor la mortalidad sobre $S$. acerifolium que sobre $S$. betaceum (Fig. 6A). No obstante, ni las épocas climáticas, las plantas hospederas o la interacción entre los factores mostraron diferencias significativas en la mortalidad de individuos (valor p. $0.33 ; 0.33 ; 0.39$, respectivamente). Las causas principales de mortalidad fueron el parasitismo, seguido de la depredación. Para las orugas, sin embargo, las hormigas fueron la causa principal de mortalidad en la época lluviosa, principalmente en cuarto estadio según la observación directa en campo, y los parasitoides en la época seca. (Fig. 6B).

Se identificaron seis familias de parasitoides de los estados inmaduros de Mechanitis menapis mantineus, de los experimentos realizados en campo. Dentro del orden Hymenoptera 

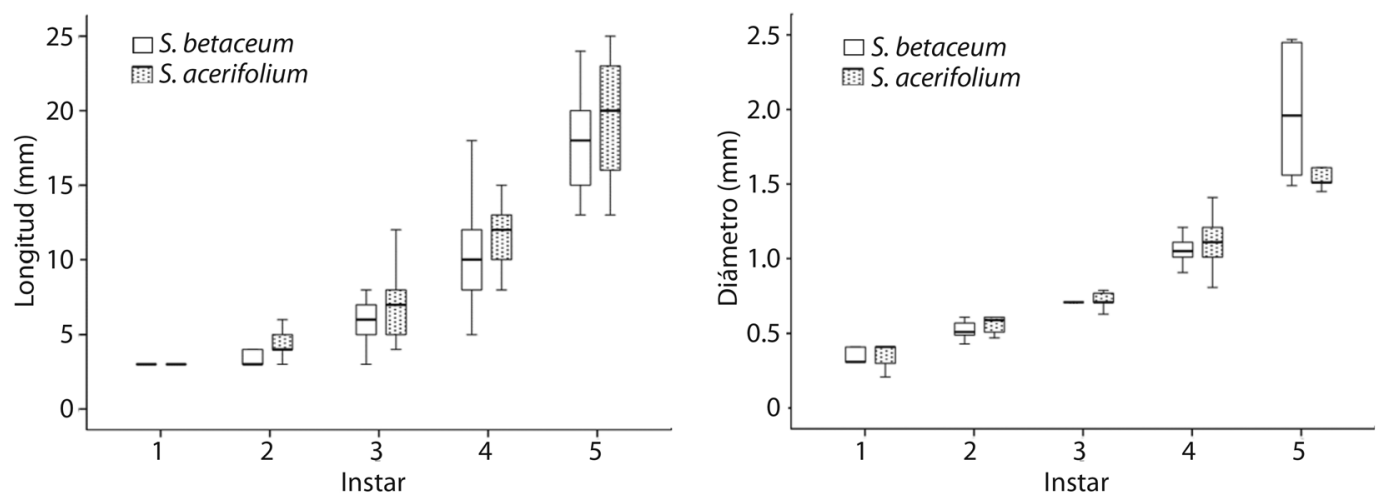

Fig. 5. Longitud del cuerpo (izquierda) y ancho de cápsulas cefálicas (derecha) de los diferentes estadios de las larvas de Mechanitis menapis mantineus en Solanum betaceum y Solanum acerifolium.

Fig. 5. Box graph for the length of the body (left) and width of head capsules (right) of the different stages of the larvae of Mechanitis menapis mantineus in Solanum betaceum and Solanum acerifolium.

A

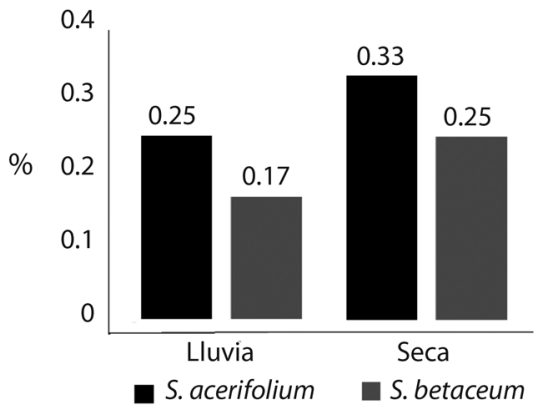

B

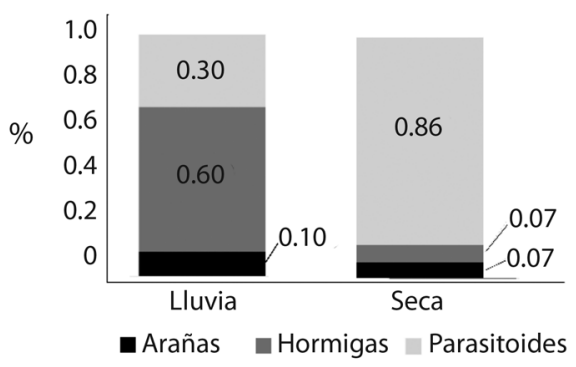

Fig. 6. Porcentaje de mortalidad de dos cohortes de orugas de Mechanitis menapis mantineus in situ, en época seca y de lluvia A) en Solanum acerifolium y Solanum betaceum; B) por diferentes agentes de mortalidad.

Fig. 6. Mortality percentage of two caterpillar cohorts of Mechanitis menapis mantineus in situ, during the dry and rainy season A) in Solanum acerifolium and Solanum betaceum; B) by different mortality agents.

se identificaron las familias Braconidae, Eulophidae, Ichneumonidae y Chalcididae (Fig. 7), principalmente durante el cuarto estadio larval y en Diptera se identificaron las familias Sarcophagidae y Muscidae, en el estado de pupa. El mayor porcentaje de mortalidad fue causado por el género Hyposoter Förster (Ichneumonidae: Campopleginae), del cual se describieron aspectos de su comportamiento reproductivo.

\section{Comportamiento del parasitoide Hypo- soter $\mathbf{s p .}$ (Ichneumonidae).}

Cortejo y cópula: El macho se aproxima volando a una hembra en reposo, mientras la hembra espera con sus antenas elevadas. A continuación, el macho palpa el sustrato con sus antenas sin desplazarse, a lo cual la hembra responde acicalándose el gáster con sus patas posteriores. Posteriormente, el macho comienza un movimiento ascendente y descendente de sus alas, mientras la hembra continúa el acicalamiento; el macho agita sus antenas sobre el cuerpo de la hembra sin hacer contacto mientras la hembra se acicala. Finalmente, el macho hace contacto con el gáster de la hembra usando sus antenas. En caso de ser receptiva, la hembra dirige sus antenas al sustrato y prosigue la cópula; caso contrario, el macho alza el vuelo y la hembra vuelve a posición de reposo (Fig. 8A). 

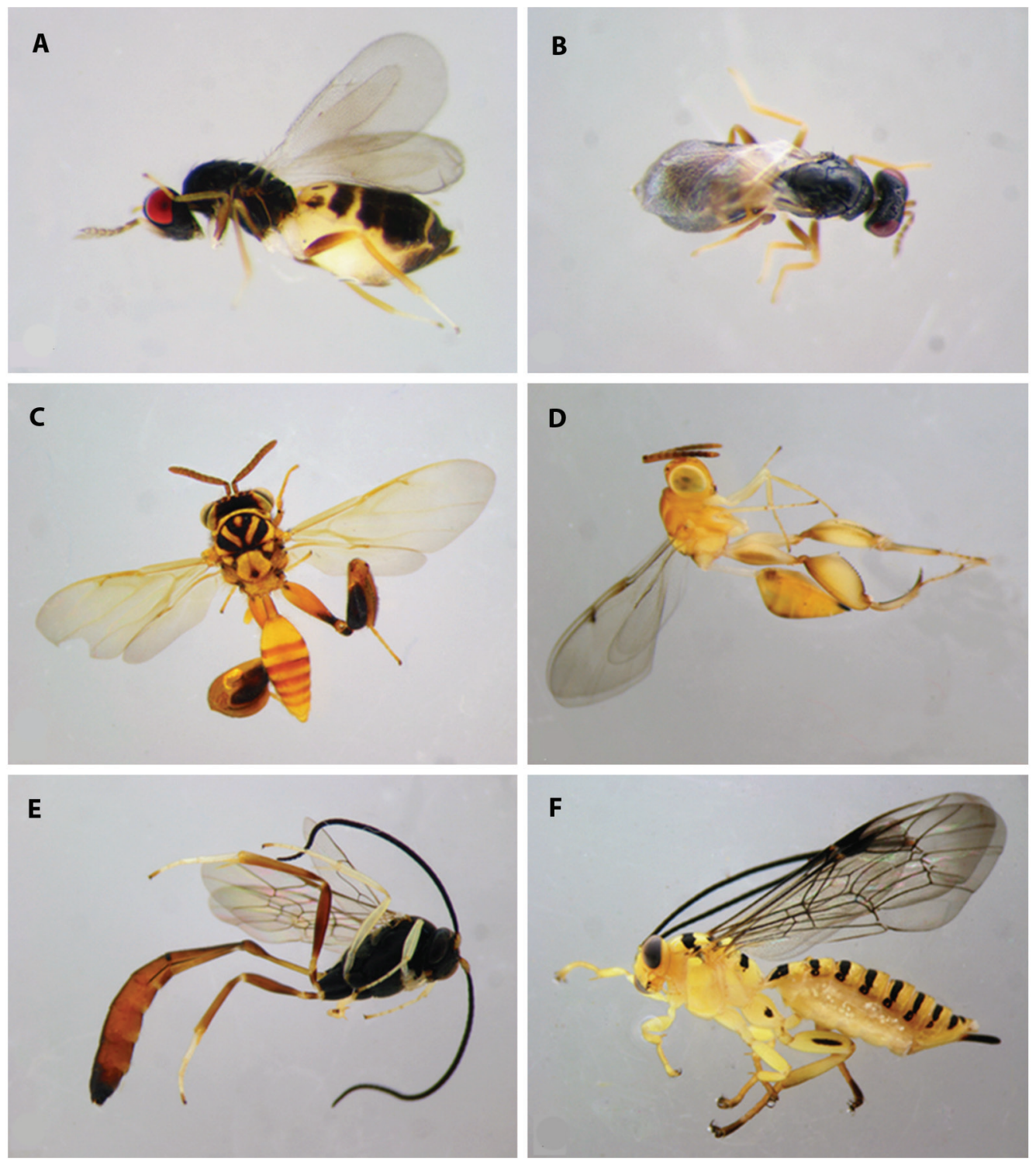

Fig. 7. Parasitoides de Mechanitis menapis mantineus: A-B (Eulophidae), de huevos; C-D (Chalcididae), de larvas; E (Ichneumonidae) y F (Braconidae), de pupa.

Fig. 7. Parasitoids of Mechanitis menapis mantineus: A-B (Eulophidae), from eggs; C-D (Chalcididae), of larvae; E (Ichneumonidae) and F (Braconidae), from pupa.

Búsqueda y oviposición: La hembra sobrevuela la planta hospedante donde se encuentran las orugas y posteriormente se posa sobre la misma; se dirige caminando sobre la hoja, con un continuo tamborileo de sus antenas, hasta las orugas. A continuación, la hembra vuela sobre la oruga y hace contacto con sus antenas.
Finalmente, la hembra introduce el ovipositor en la oruga seleccionada y realiza la oviposición, la cual se extiende por 5 segundos en promedio (Fig. 8B).

Bioensayos de contacto con la hoja de la planta hospedera: En este estudió los 


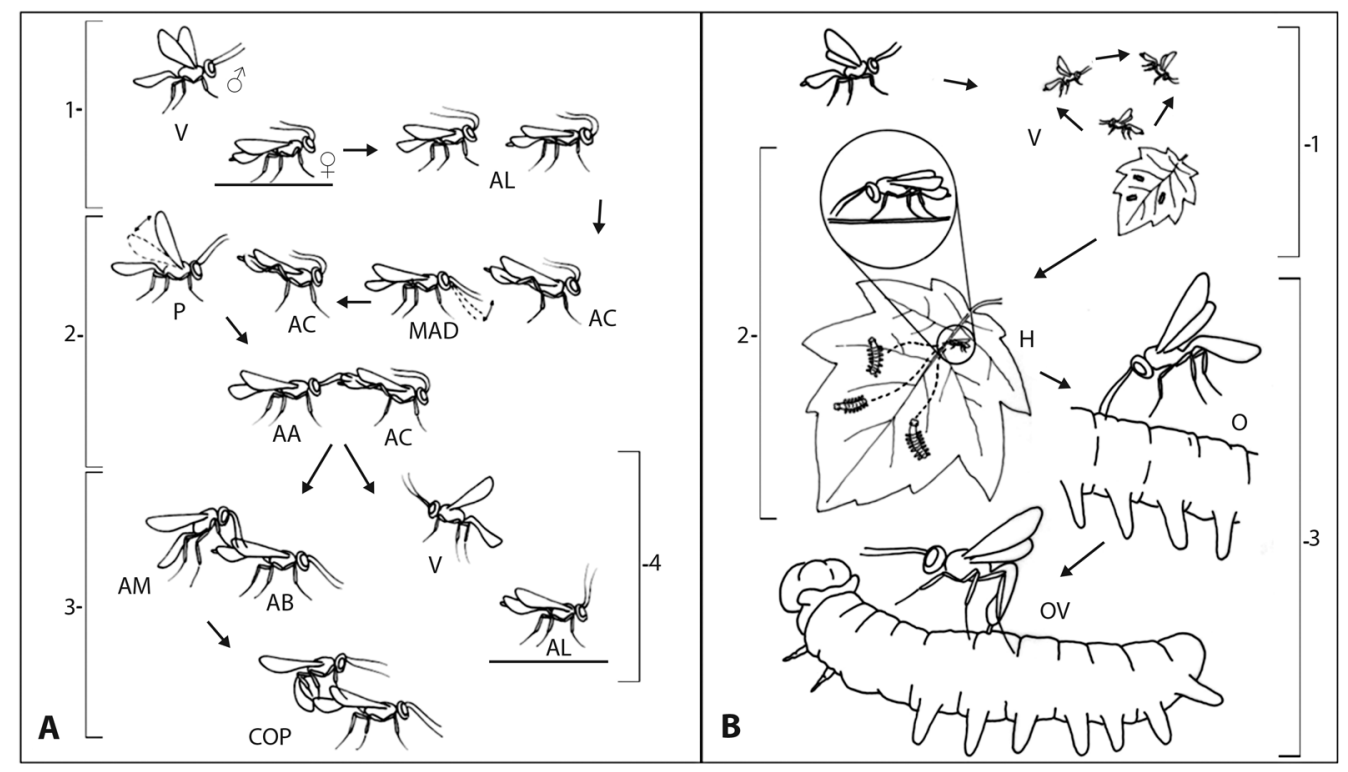

Fig. 8A. Repertorio de cortejo y cópula del parasitoide Hyposoter sp. 1.| Aproximación: Macho volando (V), hembra en reposo (AL); Macho y hembra con antenas levantadas (AL). 2. Cortejo: Macho palpa el sustrato sin desplazarse (MAD); hembra en acicalamiento (AC); macho mueve sus alas sin desplazarse (P); hembra en acicalamiento (AC); macho agita antenas sobre la hembra sin hacer contacto (AA); en acicalamiento (AC); macho toca el gáster de la hembra con sus antenas (AM); hembra (receptiva), dirige antenas hacia el sustrato (AB). 3. Cópula (COP). 4. Rechazo: Macho se retira (V), hembra (no receptiva), con antenas levantadas (AL).

Fig. 8B. Etograma de oviposición de Hyposoter sp. 1. Aproximación: Hembra sobrevolando la planta hospedera (V); tamborileo continuo de antenas (H). 2. Selección: Hembra vuela sobre la oruga y le hace contacto con sus antenas (O). 3. Oviposición: Hembra introduce el ovipositor en la oruga seleccionada (OV).

Fig. 8A. Courtship and copulation repertoire of the Hyposoter sp. 1 Approach: Flying male (V), resting female (AL); Male and female with raised antennas (AL). 2 Courtship: Male palpates the substrate without moving (MAD); grooming female (AC); male moves his wings without moving (P); grooming female (AC); male waves antennas on the female without making contact (AA); in grooming (AC); male touches the female's gaster with its antennae (AM); female (receptive), directs antennas towards the substrate $(A B)$. 3. Copulation (COP). 4. Rejection: Male withdraws (V), female (not receptive), with raised antennas (AL).

Fig. 8B. Oviposition ethogram of Hyposoter sp. 1 Approach: Female flying over the host plant (V); continuous drumming of antennas (H). 2 Selection: Female flies over the caterpillar and makes contact with its antennae (O). 3 Oviposition: Female introduces the ovipositor into the selected caterpillar (OV).

resultados del test exacto de Fisher para el experimento realizado con las escamas de la mariposa, no mostró diferencias significativas entre las dos especies hospedantes, al encontrarse por separado $(\mathrm{P}=0.085)$, ni cuando se dispusieron conjuntamente $(\mathrm{P}=0.328)$. En el experimento que incluyó tanto el excremento de oruga y las hojas con daño mecánico de las larvas, presentó diferencias significativas para las plantas hospederas separadas $(\mathrm{P}=0.02)$, con preferencia por la planta nativa $S$. acerifolium, pero no cuando ambas hospederas están juntas $(P=0.089)$. Finalmente, el experimento no mostró diferencias significativas cuando fue colocada la oruga sobre las hojas de cada una de las plantas hospederas.

\section{DISCUSIÓN}

Las plantas hospedantes aquí identificadas, son todas nuevos registros para la subespecie M. menapis mantineus. Entre ellas, S. acerifolium, es un registro común para la subespecie Mechanitis menapis doryssus Bates, 
1864 en México (Giraldo et al., 2014) y para Mechanitis menapis menapis Hewitson, [1856] en los valles interandinos colombianos (Muriel, Montoya, Restrepo, \& Muñoz, 2011). Por otra parte, $S$. betaceum es común sólo para la subespecie centroamericana (Giraldo et al., 2014) y no para los valles interandinos de Colombia, en donde no se tienen registros de esta planta sirviendo como alimento para ninguna de las especies de Mechanitis que allí ocurren. Es de notar que esta planta es ampliamente cultivada en Colombia por encima de los 1800 m.s.n.m., $y$, aunque introducida (Bohs, 2014), crece de forma silvestre en la zona de transición del bosque húmedo en México. En Mindo-Ecuador, la planta no parece crecer de forma silvestre, sin embargo, aparece ocasionalmente en huertas o jardines caseros de la zona urbana y periurbana. Finalmente, $S$. quitoense es común para la subespecie colombiana, no obstante, allí se desarrollan los individuos hasta el estado adulto según observaciones de CEG, diferente a lo encontrado en este trabajo, donde la mortalidad sobre esta planta fue del $100 \%$. La oferta y uso de plantas hospederas en los valles interandinos colombianos parece ser mucho mayor que en el pacífico ecuatoriano y en la zona de transición de mexicana. No obstante, en estas zonas, $S$. betaceum sirve como fuente alterna de alimentación para las larvas, aun cuando no se considera nativo en ninguna de las dos regiones.

Al respecto del comportamiento de oviposición, se destaca el hecho que presente algunas variaciones interesantes. Las hembras de $M$. menapis mantineus, depositan sus huevos en el haz de las hojas de sus plantas hospederas, de manera similar a M. menapis doryssus. En tres años de observaciones realizadas en este trabajo, sólo una única postura de dos huevos se encontró sobre el tallo de $S$. acerifolium. Este comportamiento parece diferir con $M$. menapis menapis, la cual deposita sus huevos tanto en el haz como el envés de las hojas (Giraldo \& Uribe, 2012), siendo más frecuente en el envés de la hoja, para registros por encima de 1400 metros de altitud (Muriel et al., 2011). Esta información es importante, para futuras exploraciones sobre alimentación y mecanismos de defensa físicos de las plantas contra sus defoliadores.

Sin embargo, el número de huevos por postura de $M$. menapis mantineus, parece no ser afectado por las plantas hospedantes que usa en la región. Lo anterior, concuerda con reportado para $M$. menapis menapis (Muriel et al., 2011; Giraldo \& Uribe, 2012) pero difiere a lo sugerido por Vasconcellos-Neto y Monteiro (1993) para Mechanitis lysimnia (Fabricius, 1793) en el sureste de Brasil. No obstante, es interesante que la cantidad de huevos por postura aquí encontrada $(1.91 \pm 1.73 ; n=409)$, parecen ser similares con los de $M$. menapis menapis en los valles interandinos en altitudes por debajo de los 1000 m.s.n.m. (2.76 \pm 2.18 ; $\mathrm{n}=104$ ) (Giraldo et al., 2014), pero no con las posturas encontradas a mayores altitudes en Colombia, $11.6 \pm 1.6 ; \mathrm{n}=9$, a 1450 m.s.n.m. y $13.2 \pm 2.6 ; \mathrm{n}=5$ a 1830 m.s.n.m. (Muriel et al., 2011), y en México para M. menapis doryssus ( $7 \pm 2.4$ huevos), a una altitud de 1400 m.s.n.m (Giraldo et al., 2014).

El gregarismo provee ventajas para los huevos y para las orugas, siendo la termorregulación, una de las más importantes en las zonas templadas (Stamp, 1980). En condiciones de cautiverio, PGS observó que distintas hembras de M. menapis mantineus depositaron sus huevos de forma aledaña en las mismas hojas, haciendo indistinguibles las distintas posturas en un grupo de ocho huevos. Este comportamiento fue reportado para Mamestra configurata Walker, 1856 (Lepidoptera: Noctuidae) (Ulmer, Gillott, \& Erlandson, 2003), pero no ha sido reportado previamente para ninguna otra especie del género Mechanitis ni de la tribu Ithomiini, a diferencia de la agregación de las larvas, registrada para la especie recientemente (Despland \& Santacruz, 2016). Así pues, se requieren observaciones adicionales en campo para corroborar que las hembras adultas de $M$. menapis favorecen el gregarismo de las larvas en campo como estrategia ecológica para incrementar la supervivencia de su descendencia.

El costo y beneficio del gregarismo para los insectos se ha debatido por mucho tiempo, sin embargo, el beneficio reconocido para la 
mayoría de grupos taxonómicos, es la protección contra los enemigos naturales (Cornell, Hawkins, \& Hochberg, 1998; Vulinec, 1990). Los individuos que viven en grupos se pueden beneficiar al incrementar las oportunidades para detectar depredadores y defenderse en conjunto (Stamp, 1980); o por el efecto de dilución o distribución del riesgo, a medida que el grupo crece, el riesgo per capita por ataques, decrece (Turchin \& Kareiva, 1989). Sin embargo, existen otros beneficios, presentes en los herbívoros, que incluyen la ubicación de pareja de pareja (Casey \& Knapp, 1987) que no beneficiaría directamente a $M$. menapis mantineus, dado el parasitismo se presenta durante su estado larval.

Finalmente, la facilidad en el forrajeo o termorregulación, podrían también reducir la depredación, al reducir el tiempo de duración de exposición de las larvas a los enemigos (Benrey \& Denno, 1997). La termorregulación podría ser relevante en la región neotropical andina, donde la temperatura disminuye con la altitud y podría afectar el comportamiento de agregación de las larvas y de oviposición de las hembras, no obstante, se requieren trabajos experimentales que soporten esta hipótesis. Finalmente, los efectos del gregarismo sobre el forrajeo, evitando el consumo de los tricomas de la plantas hospedera, fueron expuestos para M. polymnia varias décadas atrás (Rathcke \& Poole, 1975). Esta idea fue soportada en trabajos recientes, donde se sugiere que la producción de seda facilita la alimentación y ayuda a mantener la cohesión del grupo larvario (Despland \& Santacruz, 2016).

Los resultados de este trabajo, sugieren que la época seca favorece el parasitoidismo por encima de la depredación la cual, por su parte, se ve favorecida con la época lluviosa, principalmente por hormigas depredando huevos. En este sentido, no son muchos los trabajos realizados en el Neotrópico acerca de la interacción planta-hospedero-parasitoide (Moraes, Lewis, \& Tumlinson, 2000; Marconato, Dias, \& Penteado-Dias, 2008; Fernandes, Dias Filho, Fernandes, \& Penteado-Dias, 2010; Kaminski et al., 2010), y menos aún los relacionados con la tribu Ithominii. En el trabajo realizado por Muriel, Muñoz y Restrepo (2014), se describen algunos de los enemigos naturales de estas mariposas, coincidiendo con las familias de parasitoides aquí identificadas para M. menapis mantineus. No obstante, no existe información publicada sobre el comportamiento reproductivo y de apareamiento para especies de la familia Campopleginae. En varios estudios dentro de la subfamilia Campopleginae, algunas especies de Hyposoter de regiones templadas del norte construye un capullo falso (Finlayson, 1966), sin embargo, en la especie aquí descrita, la pupa del parasitoide no presenta está característica y en algunos de los casos se la ha encontrada colgada del envés de la hoja. Por otra parte, otro rasgo de importancia es la coloración de la pupa del parasitoide con manchas algo claras, que según Gauld (1995), es una estrategia para evitar la depredación al semejarse al excremento de ave.

Estudios recientes que buscan comprender las causas de la selección de la planta hospedera por insectos herbívoros, han considerado las complejas interacciones existentes entre tres o más niveles tróficos (Gratton \& Welter, 1999; Hare \& Morgan, 2000; De Moraes, Mescher, \& Tumlinson, 2001). No obstante, los resultados de este trabajo, sugieren que es mucho mayor la influencia de la oruga, que de la planta en la cual se alimenta. Esto concuerda, con lo reportado para algunas especies de lepidópteros, en las cuales parece que la interacción de daño sobre su hospedero no es estrictamente necesaria para realizar la atracción de los parasitoides (Godfray, 1994). Por ejemplo, las hembras de Ostrinia nubilalis (Hübner) (Crambidae), dejan una secreción alrededor del grupo de huevos la cual es suficiente para atraer las hembras del parasitoide Trichogramma brassicae Bezdenko (Garnier-Geoffroy, Robert, Hawlitzky, \& Frerot, 1996). Este fenómeno, concuerda con el concepto de la especialización de la dieta y uso de información química en los enemigos naturales, se espera que respondan a los volátiles de la planta en general, más que a olores que son específicos para una cierta especie de planta (Steidle \& Van Loon, 2003; Vet \& Dicke, 1992). 
Sin embargo, otros trabajos han sugerido que la interacción de distintos niveles trófi$\cos$, juega un papel fundamental en la atracción del hospedero (Godfray, 1994; Lewis et al., 2002). Por ejemplo, Spodoptera exigua (Hübner) (Noctuidae) induce la emisión de una gran cantidad de terpenoides que atraen al parasitoide, poco tiempo después de atacar plantas de maíz (Turlings, McCall, Alborn, \& Tumlinson, 1993). En el estudio aquí presentado, los resultados sugieren que la planta en sí, sin daño alguno, puede también emitir señales semioquímicas suficientes para que el parasitoide se acerque. A pesar de esto, es necesario profundizar en el estudio de la relación existente entre los Ithomiini, sus parasitoides y otros agentes de mortalidad, para comprender mejor las interacciones que median en la historia de vida de las diferentes especies. En este sentido, las mariposas del género Mechanitis parecen ser un buen punto de partida para avanzar en el entendimiento de las dinámicas poblacionales los ithominos en sus distintas áreas de distribución.

Los datos aquí presentados, son una primera aproximación a la historia de vida y factores ecológicos que puedan contribuir a la comprensión de las dinámicas poblacionales y la conservación de Mechanitis menapis mantineus, subespecie endémica de Ecuador. Este taxón, habita bosques nublados altamente fragmentados, los cuales se estima ocupan sólo el $49 \%$ de su extensión original en el país, resultado de las actividades extractivas, agropecuarias y la no existencia de áreas protegidas (Ministerio del Ambiente, EcoCiencia, \& Unión Mundial para la Naturaleza (UICN), 2001). Estudios ecológicos y taxonómicos adicionales, son necesarios entonces para determinar el estatus de conservación del taxón, ante los cambios taxonómicos sugeridos en trabajos previos (Dasmahapatra et al., 2010; Giraldo \& Uribe, 2012; Hill et al., 2012).

Declaración de ética: los autores declaran que todos están de acuerdo con esta publicación y que han hecho aportes que justifican su autoría; que no hay conflicto de interés de ningún tipo; y que han cumplido con todos los requisitos y procedimientos éticos y legales pertinentes. Todas las fuentes de financiamiento se detallan plena y claramente en la sección de agradecimientos. El respectivo documento legal firmado se encuentra en los archivos de la revista.

\section{AGRADECIMIENTOS}

Agradecemos de forma especial al Museo de Invertebrados de la Pontificia Universidad Católica (QCAZ), a Tania Iza por su trabajo en campo, y a Jean Pierre Reinoso por las ilustraciones científicas.

\section{RESUMEN}

Las mariposas de la tribu Ithomiini son uno de los modelos biológicos más estudiados en años recientes en trabajos de biogeografía, taxonomía y evolución. Sin embargo, aun cuando la biología y distribución de sus especies es mejor conocida que la de otros grupos de mariposas, existen aspectos de la historia natural poco estudiados que permitirían un mejor entendimiento del comportamiento de sus poblaciones y las interacciones con su ambiente. En este trabajo, se estudió la historia natural de la mariposa, Mechanitis menapis mantineus Hewitson (Nymphalidae: Ithomiini) y sus enemigos naturales en el oeste de Ecuador. Para ello, se realizó la identificación de las plantas hospederas, se estudió el ciclo de vida y los factores de mortalidad de los estados inmaduros. Adicionalmente, se identificaron los parasitoides asociados a la especie en la zona de estudio. Se identificaron tres plantas hospederas, las cuales son nuevos registros para la especie en la región. Asimismo, se describió la morfología de los estados inmaduros y su tiempo de desarrollo. Finalmente, se identificaron los principales enemigos naturales y se describieron aspectos de la historia natural y comportamiento de Hyposoter sp. (Ichneumonidae: Campopleginae), principal parasitoide de larvas. Los resultados de este trabajo, proporcionan información esencial para la revisión taxonómica del género Mechanitis F. y para continuar el estudio de la interacción de esta especie con sus plantas hospederas y enemigos naturales.

Palabras clave: historia natural, Solanaceae, planta hospedera, Nymphalidae, parasitoidismo, Ecuador, Andes. 


\section{REFERENCIAS}

Benrey, B., \& Denno, R. (1997). The slow-growth-highmortality hypothesis: a test using the cabbage butterfly. Ecology, 78, 987-999.

Bohs, L. (2014). Solanum betaceum Cav. In Solanaceae Source. Recuperado de: http://www.nhm.ac.uk/ research-curation/research/projects/solanaceaesource/taxonomy/description-detail.jsp?spnumber $=1301$

Brown Jr., K. S. (1977). Geographical patterns of evolution in Neotropical Lepidoptera: differentiation of the species of Melinaea and Mechanitis (Nymphalidae, Ithomiinae). Systematic Entomology, 2, 161-197.

Brown, K. S. (1979). Ecologia geográfica e evolução nas florestas neotropicais: apêndices (Vol. 1). Brasil: Universidade Estadual de Campinas.

Brown Jr., K. S. (1987). Chemistry at the Solanaceae/Ithomiinae Interface. Annals of the Missouri Botanical Garden, 74, 359-397.

Brown Jr., K. S., \& Benson, W. W. (1974). Adaptive polymorphism associated with multiple Mullerian mimicry in Heliconius numata (Lepid.: Nymph.). Biotropica, 6, 205-/228.

Brown Jr., K. S., \& Freitas, A. V. L. (1994). Juvenile stages of Ithomiinae: overview and systematics (Lepidoptera: Nymphalidae). Tropical Lepidoptera, 5, 9-20.

Casey, T., \& Knapp, R. (1987). Caterpillar thermal adaptation: behavioral differences reflect metabolic thermal sensitivities. Comparative Biochemistry and Physiology, 8A, 679-682.

Chazot, N. W. S. (2016). Into the Andes: multiple independent colonizations drive montane diversity in the Neotropical clearwing butterflies Godyridina. Molecular Ecology, 25(22), 5765-5784.

Cornell, H., Hawkins, B., \& Hochberg, M. (1998). Towards an empirically-based theory of herbivore demography. Ecological Entomology, 23, 340-349.

Dasmahapatra, K. E. (2010). Mitochondrial DNA barcoding detects some species that are real, and some that are not. Molecular Ecology Resources, 10, 264-273.

De Moraes, C. M., Mescher, M. C., \& Tumlinson, J. H. (2001). Caterpillar-induced nocturnal plant volatiles repel conspecific females. Nature, 410, 577-580.

De-Silva, D. D. (2010). Molecular phylogenetics of the Neotropical butterfly subtribe Oleriina (Nymphalidae: Danainae: Ithomiini). Molecular Phylogenetics and Evolution, 55(3), 1032-1041.

De-Silva, D. L., Elias, M., Willmott, K., Mallet, J., \& Day, J. J. (2015). Diversification of clearwing butterflies with the rise of the Andes. Journal of Biogeography, 43, 44-58.
De-Silva, D. M. B. (2017). North Andean origin and diversification of the largest ithomiine butterfly genus. Scientific Reports, 7, 45966.

Despland, E., \& Santacruz, P. (2016). Silk drives aggregation of following in the neotropical caterpillar Mechanitis menapis. Physiological Entomology, 41(3), 274-280.

Drummond, B. A., \& Brown, Jr., K. S. (1987). Ithomiinae (Lepid.:Nymphalidae): Summary of know larval food plants. Annals of the Missouri Botanical Garden, 74, 341-358.

Elias, M. G. (2008). Mutualistic interactions drive ecological niche convergence in a diverse butterfly community. PLoS Biology, 6(12), 300.

Elias, M. J. B. P. (2009). Out of the Andes: patterns of diversification in clearwing butterflies. Molecular Ecology, 18(8), 1716-1729.

Fatouros, N., Bukovinszkine, G., Lucas A., Soler, R., Dicke, M., \& Hilker, M. (2005). Oviposition-induced plant cues: do they arrest Trichogramma wasps during host location. Entomologia Experimentalis et Applicata, 115, 207-215.

Fernandes, L. B., Dias Filho M. M., Fernandes, M. A., \& Penteado-Dias. M. A. (2010). Ichneumonidae (Hymenoptera) parasitoids of Lepidoptera caterpillars feeding on Croton floribundus Spreng (Euphorbiaceae). Revista Brasileria de Entomologia, 54, 263-269.

Finlayson, T. (1966). The false cocoon of Hyposoter parorgyiae (Hymenoptera: Ichneumonidae). The Canadian Entomologist, 98, 139-140.

Fox, R. (1940). A generic review of the Ihtomiinae (Lepidotera, Nymphalidae). Transactions of the American Entomolgical Society, 3, 161-207.

Fox, R. M. (1967). A Monograph of the Ithomiidae (Lepidoptera) Part III: The Tribe Mechanitini Fox. Philadelphia, U.S.A.: American Entomological Society.

Freitas, A.V. L., \& Brown Jr., K. S. (2008). Immature stages of Ithomia salapia ardea (Lepidotera: Nymphalidae, Ithomiinae). Tropical Lepidoptera, 18(1), 17-19.

Garnier-Geoffroy, F. R. (1996). Oviposition behaviour in Ostrinia nubilalis (Lep. Pyralidae) and consequences on host location and oviposition in Trichogramma brassicae (Hym. Trichogrammatidae). Entomophaga, 41, 287-299.

Garzón-Orduña, I. S. B. (2015). Incompatible ages for clearwing butterflies based on alternative secondary calibrations. Systematic Biology, 64(5), 752-767.

Gauld, I. D. (1995). Ichneumonidae. En P. E. Hanson \& I. D. Gauld (Eds.), The Hymenoptera of Costa Rica 
(pp. 390-431). Oxford, New York, U.S.A.: Oxford University Press.

Giraldo, C. E., Ramírez-Restrepo, L., Escobar, F., \& Uribe, S. (2014). Life Cycle and Host Plants of Mechanitis menapis doryssus (Danainae: Ithomiini) in Mexico. Southwestern Entomologist, 39(4), 761-772.

Giraldo, C. E., \& Uribe, S. I. (2010a). Registro de Mechanitis polymnia (Lepidoptera: Ithomiinae) en Solanum jamaicense y ciclo de vida en laboratorio. Revista Colombiana de Entomología, 36(1), 165-168.

Giraldo, C. E., \& Uribe, S. I. (2010b). Solanum hirtum as a host plant for Mechanitis menapis menapis (Lepidoptera: Ithomiinae) in Colombia. Revista Colombiana de Entomología, 36(1), 169-171.

Giraldo, C. E., \& Uribe, S. I. (2012). Taxonomy of Mechanitis (F.) (Lepidoptera: Nymphalidae) from the West Colombian Andes: an Integrative Approach. Neotropical Entomology, 41(6), 472-484.

Godfray, H. C. J. (1994). Parasitoids. Behavioural and Evolutionary Ecology. Princeton, U.S.A.: Princeton University Press.

Gratton, C., \& Welter, S. C. (1999). Does "enemy-free space" exist? Experimental host shifts of an herbivorous fly. Ecology, 80, 773-785.

Hare, J. D., \& Morgan, D. J. W. (2000). Chemical conspicuosness of an herbivore to its natural enemy: effect of feeding site selection. Ecology, 81, 509-519.

Hill, R. I. (2012). Ecologically relevant cryptic species in the highly polymorphic Amazonian butterfly Mechanitis mazaeus sl (Lepidoptera: Nymphalidae; Ithomiini). Biological Journal of the Linnean Society, 106(3), 540-560.

Joron, M., \& Mallet, J. (1998). Diversity in mimicry: paradox or paradigm? Trends in Ecology \& Evolution, 13(11), 461-466.

Kaminski, L. (2010). Interaction between Mutualisms: Ant-Tended Butterflies. The American Naturalist, 176(3), 322-334.

Lamas, G. (2004). Checklist: Part 4 Hesperioidea-Papilionoidea (in) Atlas of Neotropical Lepidoptera. Florida: Association for Tropical Lepidoptera.

Lewis, O., Memmott, J., Lasalle, J., Lyal, C. H. C., Whitefoord, C., \& Godfray, C. (2002). Structure of a diverse tropical forest insect-parasitoid community. Journal of Animal Ecology, 71, 885-873.

Marconato, G., Dias, M. M., \& Penteado-Dias, A. M. (2008). Larvas de Geometridae (Lepidoptera) e seus parasitoides, associadas a Erythroxylum microphyllum. St. Hilaire (Erythroxylaceae). Revista Brasileira de Entomologia, 52, 296-299.

Ministerio del Ambiente, EcoCiencia \& Unión Mundial para la Naturaleza (UICN). (2001). La Biodiversidad del Ecuador (Informe 2000). C. Josse (Ed.). Quito: Ecociencia.

Moraes, C., Lewis, W. J., \& Tumlinson, J. H. (2000). Examining plant-parasitoid interactions in tritrophic systems. Annual Society Entomology of Brazil, 29, 189-203.

Muriel, S. B., Montoya, J., Restrepo, A., \& Muñoz, J. (2011). Nuevos registros de plantas hospederas y disponibilidad de recursos para mariposas Ithomiini (Lepidoptera: Nymphalidae: Danainae), en agroecosistemas de café colombianos. Actualidades Biológicas, 33(95), 275-285.

Muriel, S. B., Muñoz, J., \& Restrepo, A. (2014). Parasitoidismo de dos especies de mariposas en dos sistemas de producción de café. Revista Colombiana de Entomología, 40(2), 251-258.

Rathcke, B. J., \& Poole, R. W. (1975). Coevolutionary race continues: butterfly larval adaptation to plant trichomes. Science, 187, 175-176.

Sierra, R. (Ed.). (1999). Propuesta preliminar de un sistema de clasificación de vegetación para el Ecuador continental. Quito, Ecuador: Proyecto INEFAN/GEF/ BIRF y EcoCiencia.

Stamp, N. E. (1980). Egg deposition patterns in butterflies: why do some species cluster their eggs rather than deposit them singly. The American Naturalist, 115, 367-380.

Stehr, F. W. (1987). Immature insects. Dubuque, US: Kendall/Hunt Publishing.

Steidle, J. L. M., \& Van Loon, J. J. A. (2003). Dietary specialization and infochemical use in carnivorous arthropods: testing a concept. Entomologia Experimentalis et Applicata, 108, 133-148.

Turchin, P., \& Kareiva, P. (1989). Aggregation in Aphis varians: an effective strategy for reducing predation risk. Ecology, 70, 1008-1016.

Turlings, T. C. J., McCall, P. J., Alborn, H. T., \& Tumlinson, J. H. (1993). An Elicitor in Caterpillar Oral Secretions That Induces Corn Seedlings to Emit Chemical Signals Attractive to Parasitic Wasps. Journal of Chemical Ecology, 19(3), 411-425.

Ulmer, B., Gillott, C., \& Erlandson, M. (2003). Conspecific eggs and bertha Armyworm, Mamestra configurata (Lepidotera: Noctuidae), Oviposition Site Selection. Environmental Entomology, 32(3), 529-534. 
Vasconcellos-Neto, J., \& Monteiro, R. F. (1993). Inspection and evaluation of host plant by the butterfly Mechanitis lysimnia (Nymph., Ithomiinae) before laying eggs: a mechanism to reduce intraspecific competition Oecologia, 95(3), 431-438.

Vet, L., \& Dicke, M. (1992). Ecology of infochemical use by natural enemies in a tritrophic context. Annual Review of Entomology, 37, 141-172.

Vulinec, K. (1990). Collective security: aggregation by insects as a defense. En D. L. Evans (Ed.), Insect defenses (pp. 251-288). New York: State University of New York.

Willmott, K. R., \& Freitas, A. V. L. (2006). Higher-level phylogeny of the Ithomiinae (Lepidoptera: Nymphalidae): classification, patterns of larval hostplant colonization and diversification. Cladistics, 22(4), 297-368.

Willmott, K. R., \& Mallet, J. (2004). Correlations between adult mimicry and larval host plants in Ithomiine butterflies. Proceedings of the Royal Society B(Suppl.), 271, S266-S269. 